\title{
Robust Variance Constrained Filter Design for Systems with Non-Gaussian Noises
}

\author{
Fuwen Yang, Yongmin $\mathrm{Li}$, and Xiaohui Liu
}

\begin{abstract}
In this paper, a variance constrained filtering problem is considered for systems with both non-Gaussian noises and polytopic uncertainty. A novel filter is developed to estimate the systems states based on the current observation and known deterministic input signals. A free parameter is introduced in the filter to handle the uncertain input matrix in the known deterministic input term. In addition, unlike the existing variance constrained filters, which are constructed by the previous observation, the filter is formed from the current observation. A time-varying linear matrix inequality (LMI) approach is used to derive an upper bound of the state estimation error variance. The optimal bound is obtained by solving a convex optimisation problem via Semi-Definite Programming (SDP) approach. Simulation results are provided to demonstrate the effectiveness of the proposed method.
\end{abstract}

\section{INTRODUCTION}

Kalman filter has been widely applied in many engineering and information systems, for instance, target tracking, image processing, signal processing, communication and control engineering [1]. However, filtering performance may deteriorate by use of standard Kalman filter when the underlying systems contain parameter uncertainties and non-Gaussian noises due to unmodelled dynamics, parameter variations, model reduction, linearisation and external severe environment [25]. There are essentially two approaches to cope with parameter uncertainties and/or non-Gaussian noises. One is robust filtering and the other is $H_{\infty}$ filtering. $H_{\infty}$ filtering method provides an energy bounded gain from the noise inputs to the estimation error without the need for knowledge of noise statistics [24]. In this filtering, process and measurement noises are assumed to be arbitrary rather than Gaussian processes. It has been proven that $H_{\infty}$ filtering is less sensitive to parameter uncertainties and non-Gaussian noises, but its design is too conservative and there is no provision to ensure that the variance of the state estimation error lies within acceptable bounds [21], [24]. Robust filtering has attempted to constrain the variance in spite of large parameter uncertainties [6], [7], [11], [14], [15].

This work has been supported by the Engineering and Physical Sciences Research Council (EPSRC) of the U.K. under Grant EP/C007654/1.

F. Yang, Y. Li and X. Liu are with Department of Information Systems and Computing, Brunel University, Uxbridge, Middlesex, UB8 3PH, United Kingdom. Email: \{Fuwen.Yang, Yongmin.Li, Xiaohui.Liu\}@brunel.ac.uk, Telephone: 00441895 265439, Fax: 00441895251686.
There have been a number of literature to address the robust filtering problems with variance constrained. The robust variance constrained filtering problems were considered for linear systems with norm-bounded parameter uncertainties [18], [23]. The filter was obtained by solving two Riccatilike equations, where a scaling parameter will be searched to find a feasible solution [5], [28]. In order to avoid the scaling parameter search, an LMI approach has been applied to solve for linear systems with both norm-bounded parameter uncertainties and polytopic uncertainties [5], [16], [22]. Recently, this problem has been extensively studied. For example, the robust variance constrained filtering problem for uncertain systems with multiplicative noises has been considered in [19], [25]; the robust variance constrained filtering problem for uncertain systems with stochastic nonlinearities has been studied in [26]; the robust variance constrained filtering problem for uncertain systems with random sensor delays has been solved in [27]; the robust variance constrained filtering problem for uncertain systems with missing measurements has been investigated in [20]. So far, to the best of our knowledge, it is always assumed that the process noises and measurement noises are Gaussian white ones in the existing literature about robust variance constrained filtering. However, in practical applications, the process noises and measurement noises may be non-Gaussian [8], [9]. This motivates us to investigate the robust variance constrained filter design problems for non-Gaussian noises. Moreover, we will also investigate filtering problem for uncertain systems containing known deterministic input [10]. In deterministic systems, the existence of a known deterministic input is of no significance for the filtering problem, as the filter can be designed to cancel the effect on the estimation error. When there exist parameter uncertainties in systems, the known deterministic input will produce an unknown bias in filtering error [10]. In order to avoid the design difficulty, most of the existing works in this area assume that the system is driven only by noise processes without the presence of a known deterministic input [5]-[7], [11], [14]-[16], [18]-[20], [22], [23], [25]-[28].

In this paper, we will present several novel techniques to tackle these two problems. We combine them as a robust variance constrained filtering problem for uncertain system with known deterministic input and non-Gaussian noises. In 
particular, a novel filter is proposed based on the current observation and known deterministic input signals. A free parameter is introduced in the filter to handle the uncertain input matrix in the known deterministic input term. In addition, unlike the existing variance constrained filters, which are constructed by the previous observation, the filter is formed from the current observation. An upper bound of the state estimation error variance is derived from the system equation and the filter equation, which is an inequality containing non-Gaussian process and measurement noises constraints. $S$-procedure and Schur complement techniques are employed to combine all inequalities into a time-varying LMI. The convex combination approach is applied to handle the polytopic uncertainties in the LMI. Finally, the filtering problem is transferred into a convex optimisation problem, which is easily solved via SDP approach.

The remainder of this paper is organised as follows. Section II discusses the problem of the robust variance constrained filtering for polytopic uncertain discrete-time systems with non-Gaussian noises and known deterministic input, and Section III develops a novel algorithm to minimise the upper bound of the state estimation error variance. Section IV provides an illustrative example to demonstrate the effectiveness of our algorithm. Conclusions are drawn in Section V.

Notation. The notation $X \geq Y$ (respectively, $X>Y$ ) where $X$ and $Y$ are symmetric matrices, means that $X-Y$ is positive semi-definite (respectively, positive definite). The superscript $T$ stands for matrix transposition. The notation $\operatorname{trace}(P)$ denotes the trace of $P$.

\section{PRoBlem Formulation}

Consider the following discrete-time polytopic uncertain system:

$$
\begin{aligned}
x_{k+1} & =A_{k}(\alpha) x_{k}+F_{k}(\alpha) u_{k}+B_{k}(\alpha) w_{k}, \\
y_{k} & =C_{k} x_{k}+D_{k} v_{k},
\end{aligned}
$$

where $x_{k} \in \mathbb{R}^{n}$ is the system state, $u_{k} \in \mathbb{R}^{l}$ is the known deterministic input, $y_{k} \in \mathbb{R}^{m}$ is the measurement output, $w_{k} \in \mathbb{R}^{r}$ is the process noise, and $v_{k} \in \mathbb{R}^{p}$ is the measurement noise.

$w_{k}$ and $v_{k}$ are non-Gaussian noise signals at time step $k$, which are assumed to satisfy the following constraints:

$$
\begin{gathered}
w_{k}^{T} Q_{k}^{-1} w_{k} \leq 1, \\
v_{k}^{T} R_{k}^{-1} v_{k} \leq 1,
\end{gathered}
$$

where $Q_{k}=Q_{k}^{T}>0$ and $R_{k}=R_{k}^{T}>0$ are known matrices with compatible dimensions. The initial state $x_{0}$ is also assumed to satisfy a constraint:

$$
\left(x_{0}-\hat{x}_{0}\right)\left(x_{0}-\hat{x}_{0}\right)^{T} \leq P_{0},
$$

where $\hat{x}_{0}$ is an estimate of $x_{0}$ which is assumed to be given, and $P_{0}=P_{0}^{T}>0$ is a known matrix.

The matrices $A_{k}(\alpha), B_{k}(\alpha)$ and $F_{k}(\alpha)$ are unknown timevarying parameters with appropriate dimensions. We assume that $\left(A_{k}(\alpha), B_{k}(\alpha), F_{k}(\alpha)\right) \in \Omega$, where $\Omega$ is a convex polyhedral set described by $K$ vertices

$$
\begin{aligned}
\Omega & =\left\{\left(A_{k}(\alpha), B_{k}(\alpha), F_{k}(\alpha)\right)\right. \\
& \left.=\sum_{i=1}^{K} \alpha_{i}\left(A_{k}^{(i)}, B_{k}^{(i)}, F_{k}^{(i)}\right), \sum_{i=1}^{K} \alpha_{i}=1, \alpha_{i} \geq 0\right\},(6)
\end{aligned}
$$

where $\left(A_{k}^{(i)}, B_{k}^{(i)}, F_{k}^{(i)}\right)$ are known for all $i=1,2, \ldots, K$.

Remark 1: As compared with the norm-bounded uncertainty, the polytopic uncertainty considered in this paper is more flexible. Polytopic uncertainty is probably the most general way of capturing the structured uncertainty that may affect the system parameters. It includes the well-known interval parametric uncertainty [2].

In this paper, a novel filter based on the current observation is developed for the uncertain system (1)-(2). The filter is given in the following form:

$$
\hat{x}_{k+1}=G_{k} \hat{x}_{k}+H_{k} u_{k}+L_{k} y_{k+1},
$$

where $\hat{x}_{k} \in \mathbb{R}^{n}$ is the state estimate of $x_{k}, G_{k}, H_{k}$ and $L_{k}$ are the filter parameters to be determined.

Remark 2: The filter (7) has two remarkable advantages. The first one is to use a design parameter $H_{k}$ to cope with the uncertain input matrix $F_{k}(\alpha)$, since we cannot simply choose any matrix from the uncertain matrix $F_{k}(\alpha)$ like a fixed input matrix $F_{k}$ [10]. The second advantage is that the filter is based on the current observation $y_{k+1}$ and makes full use of the observation information. It is worth mentioning that most literature published recently construct the filter based on the previous observation $y_{k}$, for example, [5]-[7], [14][16], [18], [19], [22], [23], [28], to name a few.

Our objective is first to design the filter (7) such that an upper bound for the estimation error variance is guaranteed for all unknown matrices $\left(A_{k}(\alpha), B_{k}(\alpha), F_{k}(\alpha)\right) \in \Omega$, and then minimise such a bound in the sense of the matrix trace, that is, to find the filter ( 7$)$ and a sequence of positive-definite matrices $P_{k+1}(0<k \leq N-1)$ such that

$$
\begin{gathered}
\min _{P_{k+1}, G_{k}, H_{k}, L_{k}} \operatorname{trace}\left(P_{k+1}\right) \\
\text { subject to } \\
\left(x_{k+1}-\hat{x}_{k+1}\right)\left(x_{k+1}-\hat{x}_{k+1}\right)^{T} \leq P_{k+1},
\end{gathered}
$$

and the constraints (3) and (4). This problem will be referred to as a robust variance constrained filter design problem.

\section{RoBUst VARIANCE CONSTRAINED FILTER DESIGN}

In this section, a robust variance constrained filter will be designed for discrete-time polytopic uncertain systems 
subject to any non-Gaussian process noise and measurement noise satisfying (3) and (4). We first consider the system (1)(2) with the known parameters $\left(A_{k}, B_{k}, C_{k}, D_{k}, F_{k}\right)$. The following theorem provides a design of the filter (7) by solving the problem (8) for the deterministic system.

Theorem 1: For the deterministic system (1)-(2), the solution to the optimisation problem (8) subject to (9) and the constraints (3), (4) is obtained by solving the following convex optimisation problem:

$$
\begin{gathered}
\min _{P_{k+1}>0, G_{k}, L_{k}, \tau_{1} \geq 0, \tau_{2} \geq 0, \tau_{3} \geq 0} \operatorname{trace}\left(P_{k+1}\right) \\
\text { subject to }
\end{gathered}
$$

$\left[\begin{array}{cc}P_{k+1} & \Pi\left(\hat{x}_{k}, u_{k}\right) \\ \Pi\left(\hat{x}_{k}, u_{k}\right)^{T} & \operatorname{diag}\left(1-\tau_{1}-\tau_{2}-\tau_{3}, \tau_{1} I, \tau_{2} Q_{k}^{-1}, \tau_{3} R_{k+1}^{-1}\right)\end{array}\right] \geq 0$

where

$$
\begin{aligned}
& \Pi\left(\hat{x}_{k}, u_{k}\right)=\left[\left(I-L_{k} C_{k+1}\right) A_{k} \hat{x}_{k}-G_{k} \hat{x}_{k}-L_{k} C_{k+1} F_{k} u_{k}\right. \\
& \left.\left(I-L_{k} C_{k+1}\right) A_{k} E_{k} \quad\left(I-L_{k} C_{k+1}\right) B_{k} \quad-L_{k} D_{k+1}\right] .
\end{aligned}
$$

and $E_{k}$ is a factorisation of $P_{k}=E_{k} E_{k}^{T}$. Moreover, the filter is given by

$$
\hat{x}_{k+1}=G_{k} \hat{x}_{k}+F_{k} u_{k}+L_{k} y_{k+1} .
$$

For polytopic uncertain systems, we cannot employ Theorem 1 to obtain an optimised upper bound of the state estimation error variance and the corresponding filter. Now we apply the convex combination approach proposed by [4], [13], [22] to cope with polytopic uncertain systems.

Theorem 2: For the polytopic uncertain system (1)-(2) which parameters reside in polytope $\Omega(6)$ with given vertices $A_{k}^{(i)}, B_{k}^{(i)}$ and $F_{k}^{(i)}(i=1,2, \cdots, K), K$ is the number of the vertices, the solution to the optimisation problem (8) subject to (9) and the constraints (3), (4) is obtained by solving the following convex optimisation problem:

$$
\begin{gathered}
\min _{P_{k+1}>0, G_{k}, H_{k}, L_{k}, \tau_{1} \geq 0, \tau_{2} \geq 0, \tau_{3} \geq 0} \operatorname{trace}\left(P_{k+1}\right) \\
\text { subject to } \\
{\left[\begin{array}{cc}
P_{k+1} & \Pi^{(i)}\left(\hat{x}_{k}, u_{k}\right) \\
\Pi^{(i)}\left(\hat{x}_{k}, u_{k}\right)^{T} & \operatorname{diag}\left(1-\tau_{1}-\tau_{2}-\tau_{3}, \tau_{1} I, \tau_{2} Q_{k}^{-1}, \tau_{3} R_{k+1}^{-1}\right)
\end{array}\right] \geq 0}
\end{gathered}
$$

where

$$
\begin{aligned}
& \Pi^{(i)}\left(\hat{x}_{k}, u_{k}\right)=\left[\begin{array}{ll}
\Pi_{1}^{(i)}\left(\hat{x}_{k}, u_{k}\right) & \left(I-L_{k} C_{k+1}\right) A_{k}^{(i)} E_{k} \\
& \left(I-L_{k} C_{k+1}\right) B_{k}^{(i)}-L_{k} D_{k+1}
\end{array}\right] .(16) \\
& \\
& \Pi_{1}^{(i)}\left(\hat{x}_{k}, u_{k}\right)=\left(I-L_{k} C_{k+1}\right) A_{k}^{(i)} \hat{x}_{k}-G_{k} \hat{x}_{k}-H_{k} u_{k} \\
&+\left(I-L_{k} C_{k+1}\right) F_{k}^{(i)} u_{k},
\end{aligned}
$$

for all $i \in\{1,2, \cdots, K\}$, and $E_{k}$ is a factorisation of $P_{k}=$ $E_{k} E_{k}^{T}$. Moreover, the filter is given by

$$
\hat{x}_{k+1}=G_{k} \hat{x}_{k}+H_{k} u_{k}+L_{k} y_{k+1} .
$$

$A_{k}^{(i)}, B_{k}^{(i)}$ and $F_{k}^{(i)}$ are the matrices in (6) at the $i$ th vertex of the polytope.

Remark 3: We can see from Theorem 2 that the inequalities (15) are linear to the variables $P_{k+1}, G_{k}, H_{k}$ and $L_{k}, \tau_{1}$, $\tau_{2}, \tau_{3}$, Hence, the optimisation problems (14) can be solved by the existing SDP via interior-point approach.

\section{An Illustrative Example}

\section{Consider an uncertain system}

$$
\begin{aligned}
x_{k+1} & =\left[\begin{array}{cc}
0 & -0.95 \\
0.9+\alpha & 0.8
\end{array}\right] x_{k}+\left[\begin{array}{l}
1 \\
2
\end{array}\right] u_{k}+\left[\begin{array}{c}
10 \\
2
\end{array}\right] w_{k} \\
y_{k} & =\left[\begin{array}{ll}
0.1 & 0.5
\end{array}\right] x_{k}+v_{k}
\end{aligned}
$$

Due to modelling errors, $\alpha$ is unknown but it belongs to the known interval $\left[\alpha_{\min }, \alpha_{\max }\right]$, where $\alpha_{\min }=-0.03$ and $\alpha_{\max }=0.03$.

In the simulation, $w_{k}$ and $v_{k}$ are chosen as $0.5 \sin (2 k)$ and $0.5 \sin (30 k)$, respectively. The input is set as $u_{k}=10$. The initial state and state estimate are assumed as $x_{0}=$ $\left[\begin{array}{ll}10 & -5\end{array}\right]^{T}$ and $\hat{x}_{0}=\left[\begin{array}{ll}0 & 0\end{array}\right]^{T}$, respectively. The initial variance is assumed as $P_{0}=\left[\begin{array}{cc}90 & 0 \\ 0 & 30\end{array}\right]$. For all $k, Q_{k}=0.25$ and $R_{k}=0.25$.

The simulation results are obtained by solving the convex optimisation problem (14)-(15) in Theorem 2 under Matlab 6.5 with YALMIP 3.0 and SeDuMi 1.1 [12]. Fig. 1 and Fig. 2 show that the actual variances of the states stay below their upper bounds. Therefore the proposed design method provides an expected variance constraint. However. due to the non-Gaussian noises, the upper bounds seem too conservative. The actual state response $x_{1}$ and its estimate $\hat{x}_{1}$ generated by the filter (18) are shown in Fig. 3, and the actual state response $x_{2}$ and its estimate $\hat{x}_{2}$ are plotted in Fig. 4.

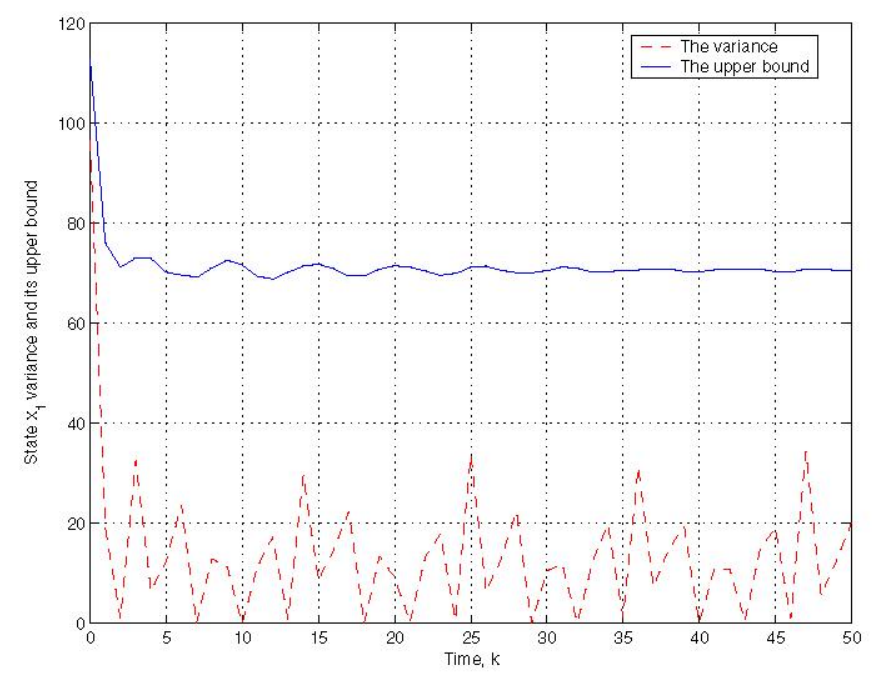

Fig. 1. The actual state $x_{1}$ estimation error variance and its upper bound. 


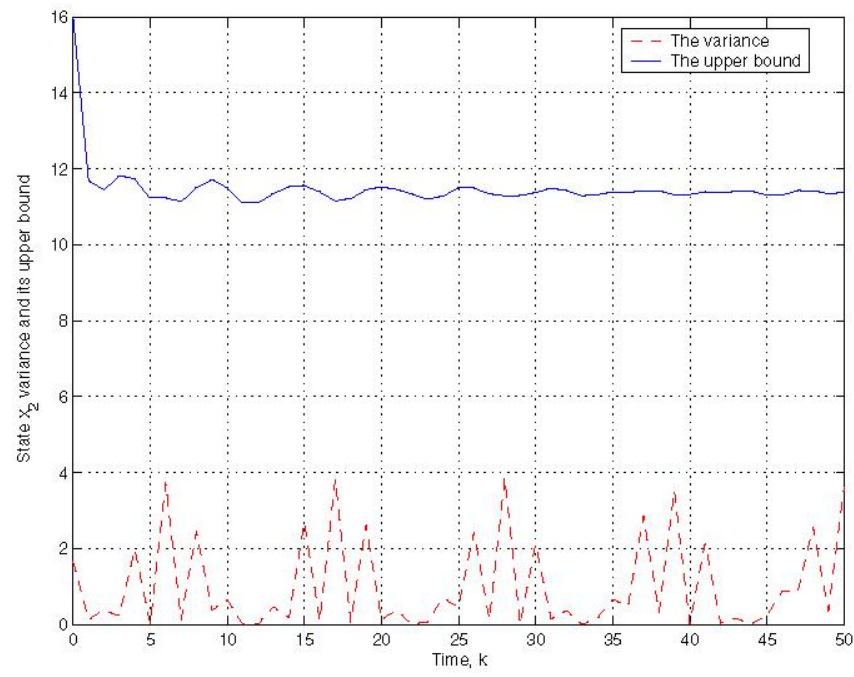

Fig. 2. The actual state $x_{2}$ estimation error variance and its upper bound.

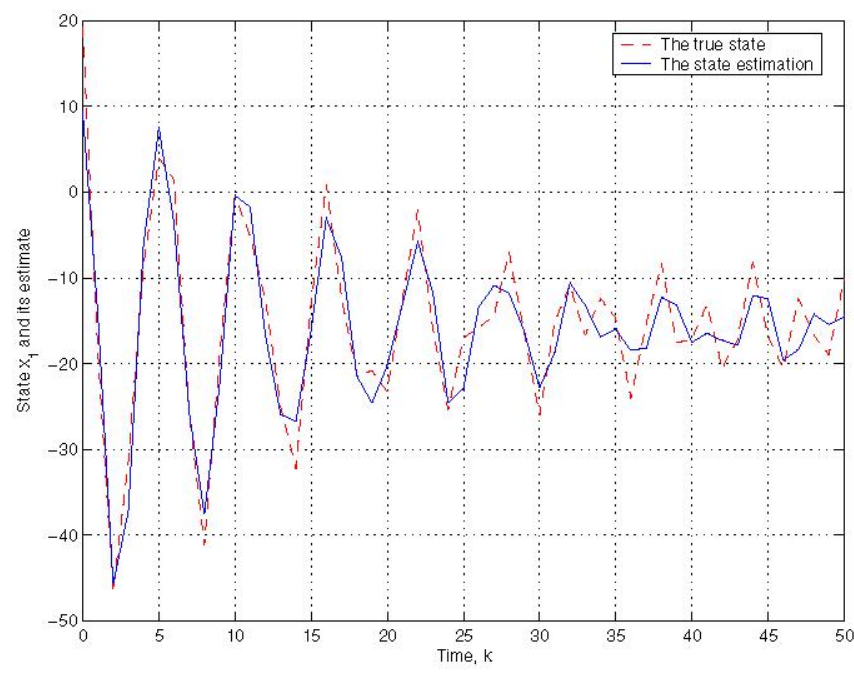

Fig. 3. The true state $x_{1}$ value and its estimate.

\section{CONCLusions}

In this paper, a variance constrained filtering problem has been considered for discrete-time systems with polytopic uncertainty and non-Gaussian noises. The proposed filter has been constructed from the current observation and known deterministic input signals. The time-varying LMI approach has been applied to derive an upper bound of the state estimation error variance which is optimised by solving a convex optimisation problem SDP approach. An illustrative example has demonstrated the feasibility of the proposed filtering methods. The filtering algorithm is computationally attractive for on-line systems with polytopic uncertainties and non-Gaussian noises.

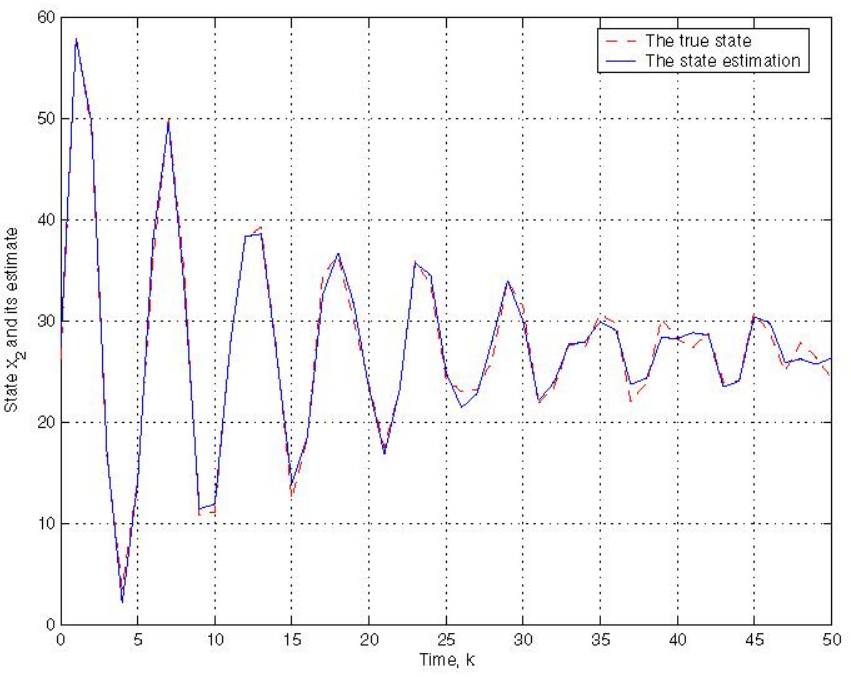

Fig. 4. The true state $x_{2}$ value and its estimate.

\section{REFERENCES}

[1] B. D. O. Anderson and J. B. Moore, Optimal Filtering. Englewood Cliffs, NJ: Prentice-Hall, 1979.

[2] S. P. Bhattacharyya, H. Chapellat, and L. H. Keel, Robust Control: The Parametric Approach. Upper Saddle River, NJ: Prentice-Hall, 1995.

[3] S. Boyd, L. E. Ghaoui, E. Feron, and V. Balakrishnan, Linear Matrix Inequalities in System and Control Theory, SIAM Studies in Applied Mathematics, Philadelphia, 1994.

[4] M. C. de Oliviera, J. Bernussou, and J. C. Geromel, "A new discretetime robust stability condition," Systems \& Control Letters, vol. 37, no. 4, pp. 261-265, 1999.

[5] M. Fu, C. E. de Souza, and Z. Luo, "Finite horizon robust Kalman filter design," IEEE Trans. Signal Processing, vol. 49, no. 9, pp. 21032112, Sept. 2001.

[6] J. C. Geromel, "Optimal linear filtering under parameter uncertainty," IEEE Trans. Signal Processing, vol. 47, no. 1, pp. 168-175, Jan. 1999.

[7] L. E1 Ghaoui and G. Calafiore, "Robust filtering for discrete-time systems with bounded noise and parametric uncertainty," IEEE Trans. Automatic Control, vol. 46, no. 7, pp. 1084-1089, July 2001.

[8] L. Guo, and H. Wang, "Fault detection and diagnosis for general stochastic systems using B-spline expansions and nonlinear observers," IEEE Trans. Circuits and Systems I, vol. 52, no. 8, pp. 1644-1652, Aug. 2005.

[9] L. Guo, and H. Wang, "PD controller design for output PDFs of stochastic systems using linear matrix inequalities," IEEE Trans. Syst., Man, Cybern. B, vol. 35, no. 1, pp. 65-71, Feb. 2005.

[10] Y. S. Hung and F. Yang, "Robust $H_{\infty}$ filtering for discrete timevarying uncertain systems with a known deterministic input," Int. $J$ of Control, vol. 75, no. 15, pp. 1159-1169, 2002.

[11] Y. S. Hung and F. Yang, "Robust $H_{\infty}$ filtering with error variance constraints for uncertain discrete time-varying systems with uncertainty," Automatica, vol. 39, no. 7, pp. 1185-1194, 2003.

[12] J. Löfberg, "YALMIP: a toolbox for modeling and optimization in Matlab," in Proceedings of the IEEE CACSD Symposium, Taipei, Taiwan, 2004.

[13] D. Peaucelle, D. Arzelier, O. Bachelier, and J. Bernussou, "A new robust D-stability condition for real convex polytopic uncertainty," Systems \& Control Letters, vol. 40, no. 1, pp. 21-30, 2000.

[14] I. Petersen and A. V. Savkin, Robust Kalman Filtering for Signals and Systems with Large Uncertainties. Series: Control Engineering, Boston: Birkhauser, 1999. 
[15] A. V. Savkin and I. R. Petersen, "Recursive state estimation for uncertain systems with an integral quadratic constraint," IEEE Trans. Alutomatic Control, vol. 40, no. 6, pp. 1080-1083, June 1995.

[16] U. Shaked, L. Xie, and Y. C. Soh, "New approaches to robust minimum variance filter design," IEEE Trans. Signal Processing, vol. 49 , no. 11, pp. 2620-2629, Nov. 2001.

[17] R. E. Skelton, T. Iwasaki, and K. Grigoriadis, A Unified Algebraic Approach to Linear Control Design. Bristol, PA: Taylor \& Francis, 1998.

[18] Y. Theodor and U. Shaked, "Robust discrete-time minimum-variance filtering," IEEE Trans. Signal Processing, vol. 44, no. 2, pp. 181-189, Feb. 1996.

[19] F. Wang and V. Balakrishnan, "Robust Kalman filters for linear time-varying systems with stochastic parametric uncertainties," IEEE Trans. Signal Processing, vol. 50, no. 4, pp. 803-813, Apr. 2002.

[20] Z. Wang, F. Yang, D. W. C. Ho, and X. Liu, "Robust finite-horizon filtering for stochastic systems with missing measurements," IEEE Signal Processing Letters, vol. 12, no. 6, pp. 437-440, 2005.

[21] Z. Wang, F. Yang, D. W. C. Ho, and X. Liu, "Robust $H_{\infty}$ filtering for stochastic time-delay systems with missing measurements," IEEE Trans. Signal Processing, vol. 54, no. 7, pp. 2579-2587, July 2006.

[22] L. Xie, L. Lu, D. Zhang, and H. Zhang, "Improved robust $H_{2}$ and $H_{\infty}$ filtering for uncertain discrete-time systems," Automatica, vol. 40, pp. 873-880, 2004

[23] L. Xie, Y. C. Soh, and C. E. de Souza, "Robust Kalman filtering for uncertain discrete-time systems," IEEE Trans. Automat. Control, vol. 39, no. 6, pp. 1310-1314, June 1994.

[24] F. Yang and Y. S. Hung, "Robust mixed $H_{2} / H_{\infty}$ filtering with regional pole assignment for uncertain discrete-time systems," IEEE Trans. Circuits and Systems-I, vol. 49, no. 8, pp. 1236-1241, Aug. 2002.

[25] F. Yang, Z. Wang, and Y. S. Hung, "Robust Kalman filtering for discrete time-varying uncertain systems with multiplicative noise," IEEE Trans. Automatic Control, vol. 47, no. 7, pp. 1179-1183, July 2002.

[26] F. Yang, Z. Wang, D. W. C. Ho, and X. Liu, "Robust $H_{2}$ filtering for a class of systems with stochastic nonlinearities," IEEE Trans. Circuits \& Systems II, vol. 53, no. 3, pp. 235-239, March 2006.

[27] F. Yang, Z. Wang, G. Feng and X. Liu, Robust filtering with randomly varying sensor delay: the finite-horizon case, Revised to IEEE Trans. Circuits and Systems I, 2007.

[28] X. Zhu, Y. C. Soh, and L. Xie, "Design and analysis of discrete-time robust Kalman filters," Automatica, vol. 38, no. 6, pp. 1069-1077, 2002. 